ISSN 1855-3966 (printed edn.), ISSN 1855-3974 (electronic edn.)

ARS MATHEMATICA CONTEMPORANEA 21 (2021) \#P1.10

https://doi.org/10.26493/1855-3974.2405.b43

(Also available at http://amc-journal.eu)

\title{
A characterization of exceptional pseudocyclic association schemes by multidimensional intersection numbers
}

\author{
Gang Chen * (D), Jiawei $\mathrm{He}^{\dagger}$ \\ School of Mathematics and Statistics, Central China Normal University, Wuhan, China \\ Ilia Ponomarenko \\ Steklov Institute of Mathematics at St. Petersburg, Russia; \\ Sobolev Institute of Mathematics, Novosibirsk, Russia; and \\ School of Mathematics and Statistics of Central China Normal University, Wuhan, China \\ Andrey Vasil'ev $§$ \\ Sobolev Institute of Mathematics, Novosibirsk, Russia; and \\ Novosibirsk State University, Novosibirsk, Russia
}

Received 10 August 2020, accepted 6 April 2021, published online 13 September 2021

\begin{abstract}
Recent classification of $\frac{3}{2}$-transitive permutation groups leaves us with three infinite families of groups which are neither 2-transitive, nor Frobenius, nor one-dimensional affine. The groups of the first two families correspond to special actions of $\operatorname{PSL}(2, q)$ and $\operatorname{P\Gamma L}(2, q)$, whereas those of the third family are the affine solvable subgroups of $\operatorname{AGL}(2, q)$ found by D. Passman in 1967. The association schemes of the groups in each of these families are known to be pseudocyclic. It is proved that apart from three particular cases, each of these exceptional pseudocyclic schemes is characterized up to isomorphism by the tensor of its 3-dimensional intersection numbers.
\end{abstract}

Keywords: Association schemes, groups, coherent configurations.

Math. Subj. Class. (2020): 05E30, 05E18

\footnotetext{
*The author was supported by the NSFC grant No. 11971189.

${ }^{\dagger}$ The author was supported by the NSFC grant No. 11971189.

¥Corresponding author. The author was supported by the Mathematical Center in Akademgorodok under agreement No. 075-15-2019-1613 with the Ministry of Science and Higher Education of the Russian Federation.

$\S$ The author was supported by the Mathematical Center in Akademgorodok under agreement No. 075-152019-1613 with the Ministry of Science and Higher Education of the Russian Federation.

E-mail addresses: chengangmath@mail.ccnu.edu.cn (Gang Chen), hjwywh@mails.ccnu.edu.cn (Jiawei He), inp@pdmi.ras.ru (Ilia Ponomarenko), vasand@math.nsc.ru (Andrey Vasil'ev)
} 


\section{Introduction}

In the late 1960s, H. Wielandt proposed a method for studying permutation groups via invariant relations. Later, D. Higman axiomatized a part of this method (connected with binary relations) by introducing a new object called a coherent configuration [6]. The coherent configuration of a permutation group $G$ is formed by the orbits of the induced action of $G$ on the Cartesian square of the underlying set of points (for exact definitions, see Section 2). Looking only at the parameters of this coherent configuration, the so-called intersection numbers, one can easily determine whether the original group is transitive, primitive, 2-transitive, etc. For example, the transitivity of a group $G$ means exactly that the coherent configuration of $G$ is an association scheme.

The concept of pseudocyclic (association) scheme goes back to research of D. Mesner [11], related with constructing of designs and strongly regular graphs; the defining property of such a scheme is that the ratio of the multiplicity and degree of its nonprincipal irreducible character does not depend on the choice of the character. It was proved in [13, Theorem 3.2] that this is equivalent to a certain relation for intersection numbers, see Subsection 2.6.

The class of pseudocyclic schemes contains all Frobenius schemes, i.e., the coherent configurations of the Frobenius groups, and, moreover, every pseudocyclic scheme of rank sufficiently large comparing with its degree is Frobenius [13, Theorem 1.1]. Thus the pseudocyclic schemes can be considered as combinatorial analogs of the Frobenius groups. It should be mentioned that the analogy is not complete, because there exist schurian (i.e., those associated with permutation groups) pseudocyclic schemes which are not Frobenius, as well as non-schurian pseudocyclic schemes [2, Example 2.6.15].

It is well known that every Frobenius group is $\frac{3}{2}$-transitive, i.e., is transitive and all the orbits of the stabilizer of a point $\alpha$, other than $\{\alpha\}$, have the same size greater than 1 . It immediately follows that so is the automorphism group of any Frobenius scheme. Moreover, the above mentioned relation for intersection numbers implies that the automorphism group of a schurian pseudocyclic scheme is also $\frac{3}{2}$-transitive. A recent classification of $\frac{3}{2}$-transitive permutation groups shows that in most cases the coherent configuration of a $\frac{3}{2}$-transitive group is pseudocyclic, see Subsection 6.1. We cite a part of this classification in the following theorem, see [10, Corollaries 2,3].

Theorem 1.1. Let $G$ be a $\frac{3}{2}$-transitive permutation group of degree $n$. Assume that neither $G$ is 2-transitive or Frobenius nor $G \leq \mathrm{A \Gamma L}(1, q)$ for some prime power $q$. Then apart from finitely many cases,

(1) $n=q(q-1) / 2, q=2^{d} \geq 8$, and either $G=\operatorname{PSL}(2, q)$, or $d$ is prime and $G=\operatorname{P\Gamma L}(2, q)$,

(2) $n=q^{2}$, $q$ is odd, and $G \leq \operatorname{AGL}(2, q)$ is the affine group with point stabilizer of order $4(q-1)$, consisting of all monomial matrices of determinant \pm 1 .

Remark 1.2. The finitely many cases mentioned in Theorem 1.1 include some affine groups of degree at most $13^{4}$ and the groups $\operatorname{Alt}(7)$ and $\operatorname{Sym}(7)$ both of degree 21.

The association schemes of the groups in statement (1) of Theorem 1.1 appeared in Master Thesis of H. Hollmann (1982); such a scheme is called a large or small Hollmann 
scheme depending on whether $G=\operatorname{PSL}(2, q)$ or $G=\operatorname{P\Gamma L}(2, q) .{ }^{1}$ These schemes have been studied in [8]; in particular, it was proved there that both are pseudocyclic. The group in statement (2) of Theorem 1.1 appeared in D. Passman's characterization of solvable $\frac{3}{2}$ transitive groups [16]. The association scheme of this group, the Passman scheme, is also pseudocyclic [13].

The goal of the present paper is to establish combinatorial characterizations of the Hollmann and Passman schemes; from the point of view of Theorem 1.1, they can naturally be considered as exceptional. One of the best possible combinatorial characterization of an association scheme is obtained when the scheme in question is determined up to (combinatorial) isomorphism by its intersection numbers; in this case the scheme is called separable. However, most of association schemes are not separable. In [4], multidimensional intersection numbers and separability number $s(\mathcal{X})$ of a coherent configuration $\mathcal{X}$ have been introduced and studied (see also [2, Section 3.5 and 4.2]). According to the definition, $s(\mathcal{X}) \leq m$ if and only if $\mathcal{X}$ is determined up to isomorphism by its $m$-dimensional intersection numbers; thus $s(\mathcal{X})=1$ if and only if $\mathcal{X}$ is separable. It was proved in [4] that $s(\mathcal{X})=1$ or 2 if $\mathcal{X}$ is the scheme of a Hamming, Johnson, or Grassmann graph; later, the estimate $s(\mathcal{X}) \leq 3$ has been established in [5] for any cyclotomic scheme $\mathcal{X}$ over finite field.

Theorem 1.3. Let $\mathcal{X}$ be a large Hollmann scheme. Then $s(\mathcal{X}) \leq 2$.

The proof of Theorem 1.3 is given in Section 3. The difficult step in the proof is to verify that the one point extension of the scheme $\mathcal{X}$ (which is a combinatorial analog of a one point stabilizer of a permutation group) is a coherent configuration of the stabilizer of this point in $\operatorname{Aut}(\mathcal{X})$. In proving this fact we use the formulas for the intersection numbers of $\mathcal{X}$, which were calculated in [8].

The proofs of the following two theorems are based on Theorem 4.1 (see Section 4), giving a sufficient condition for an arbitrary coherent configuration $\mathcal{X}$ to be partly regular, i.e., to be the coherent configuration of a permutation group having a faithful regular orbit. Using this sufficient condition we are able to show that if $\mathcal{X}$ is the small Hollmann scheme (apart from several exceptions) or the Passman scheme, then a two point extension of $\mathcal{X}$ is partly regular. Modulo known results, this immediately implies that $s(\mathcal{X}) \leq 3$.

Theorem 1.4. Let $\mathcal{X}$ be a small Hollmann scheme of degree $q(q-1) / 2$, where $q=2^{d}$ with prime $d \neq 7,11,13$. Then $s(\mathcal{X}) \leq 3$.

In the three exceptional cases of Theorem 1.4, the sufficient condition given in Theorem 4.1 does not work. It seems that the conclusion of Theorem 1.4 is also true for them. However, the corresponding schemes are too large to check this statement by a direct calculation.

Theorem 1.5. Let $\mathcal{X}$ be a Passman scheme. Then $s(\mathcal{X}) \leq 3$.

Throughout the paper, we actively use the notation, concepts, and statements from the theory of coherent configurations. All of them can be found in the monograph [2]. In Section 2, we give a brief extract from the theory of coherent configurations that is relevant for this paper.

\footnotetext{
${ }^{1}$ According to the Galois correspondence between permutation groups and coherent configurations [2, Section 2.2], the smaller groups correspond to larger coherent configurations
} 
The authors are grateful to the anonymous referees whose valuable comments helped to improve the presentation and eliminate inaccuracies, and to Saveliy Skresanov for his supplement to the GAP-package COCO2P, that was used for the computations related to Theorem 5.5.

\section{Notation.}

- For a prime power $q, \mathbb{F}_{q}$ is a finite field of order $q$.

- Throughout the paper, $\Omega$ is a finite set.

- The diagonal of the Cartesian product $\Omega \times \Omega$ is denoted by $1_{\Omega}$; for $\alpha \in \Omega$, we set $1_{\alpha}:=1_{\{\alpha\}}$.

- For $r \subseteq \Omega \times \Omega$, we set $r^{*}=\{(\beta, \alpha):(\alpha, \beta) \in r\}$ and $\alpha r=\{\beta \in \Omega:(\alpha, \beta) \in r\}$, $\alpha \in \Omega$.

- For relations $r, s \subseteq \Omega \times \Omega$, we set $r \cdot s=\{(\alpha, \beta):(\alpha, \gamma) \in r,(\gamma, \beta) \in s\}$.

- For a set $S$ of relations on $\Omega$, we define $S^{*}=\left\{s^{*}: s \in S\right\}$ and put $S^{\cup}$ to be the set of all unions of the relations of $S$.

\section{Coherent configurations}

\subsection{Rainbows}

Let $\Omega$ be a finite set and $S$ a partition of $\Omega \times \Omega$. A pair $\mathcal{X}=(\Omega, S)$ is called a rainbow on $\Omega$ if

$$
1_{\Omega} \in S^{\cup} \text {, and } S^{*}=S \text {. }
$$

The elements of the sets $\Omega, S=S(\mathcal{X})$, and $S^{\cup}$ are called, respectively, the points, basis relations, and relations of $\mathcal{X}$. The numbers $|\Omega|$ and $|S|$ are called the degree and rank of $\mathcal{X}$, respectively. The unique basis relation containing a pair $(\alpha, \beta) \in \Omega \times \Omega$ is denoted by $r_{\mathcal{X}}(\alpha, \beta)$; we omit the subscript $\mathcal{X}$ wherever it does not lead to misunderstanding.

A set $\Delta \subseteq \Omega$ is called a fiber of the rainbow $\mathcal{X}$ if $1_{\Delta} \in S$; the set of all fibers is denoted by $F:=F(\mathcal{X})$. The point set $\Omega$ is the disjoint union of fibers. If $\Delta$ is a union of fibers, then the pair

$$
\mathcal{X}_{\Delta}=\left(\Delta, S_{\Delta}\right)
$$

is a rainbow, where $S_{\Delta}$ consists of all $s_{\Delta}=s \cap(\Delta \times \Delta), s \in S$.

Let $\mathcal{X}=(\Omega, S)$ and $\mathcal{X}^{\prime}=\left(\Omega^{\prime}, S^{\prime}\right)$ be rainbows. A bijection $f: \Omega \rightarrow \Omega^{\prime}$ is called a combinatorial isomorphism (or simply isomorphism) from $\mathcal{X}$ to $\mathcal{X}^{\prime}$ if $S^{f}=S^{\prime}$, where $S^{f}=\left\{s^{f}: s \in S\right\}$. When $\mathcal{X}=\mathcal{X}^{\prime}$, the set of all these isomorphisms form a permutation group on $\Omega$. This group has a (normal) subgroup

$$
\operatorname{Aut}(\mathcal{X})=\left\{f \in \operatorname{Sym}(\Omega): s^{f}=s \text { for all } s \in S\right\},
$$

called the automorphism group of $\mathcal{X}$. 


\subsection{Coherent configurations}

A rainbow $\mathcal{X}=(\Omega, S)$ is called a coherent configuration if for any $r, s, t \in S$, the number

$$
c_{r s}^{t}=\left|\alpha r \cap \beta s^{*}\right|
$$

does not depend on the choice of $(\alpha, \beta) \in t$; the numbers $c_{r s}^{t}$ are called the intersection numbers of $\mathcal{X}$. If, in addition, $1_{\Omega} \in S$, then the coherent configuration $\mathcal{X}$ is said to be homogeneous, an association scheme, or just a scheme. A scheme $\mathcal{X}$ is called symmetric if $s=s^{*}$ for all $s \in S$.

Let $\mathcal{X}$ be a coherent configuration. Then for any $s \in S$, there exist uniquely determined $\Delta, \Gamma \in F$ such that $s \subseteq \Delta \times \Gamma$. Denote by $S_{\Delta, \Gamma}$ the set of all $s$ contained in $\Delta \times \Gamma$. Then the union

$$
S=\bigcup_{\Delta, \Gamma \in F} S_{\Delta, \Gamma}
$$

is disjoint. The positive integer $|\delta s|, \delta \in \Delta$, equals the intersection number $c_{s s^{*}}^{1_{\Delta}}$, and hence does not depend on the choice of $\delta$. It is called the valency of $s$ and denoted by $n_{s}$. In homogeneous case, $n_{s}=n_{s^{*}}$ and also

$$
n_{t} c_{r s}^{t^{*}}=n_{r} c_{s t}^{r^{*}}=n_{s} c_{t r}^{s^{*}}, \quad r, s, t \in S
$$

A basis relation $s \in S$ is called a matching if $n_{s}=n_{s^{*}}=1$; note that $s$ is not necessarily symmetric like in theory of undirected graphs. The matching $s \in S_{\Delta, \Gamma}$ defines a bijection from $\Delta$ to $\Gamma$, taking $\delta \in \Delta$ to the unique point of the singleton $\delta s$. Furthermore, one can see that if $r \in S$ and $t=s \cdot r$ (respectively, $t=r \cdot s$ ) is nonempty, then $t \in S$.

Let $G$ be a permutation group on $\Omega$. Denote by $(\alpha, \beta)^{G}$ the orbit of the induced action of $G$ on $\Omega \times \Omega$, that contains the pair $(\alpha, \beta)$. Then

$$
\operatorname{Inv}(G)=\operatorname{Inv}(G, \Omega)=\left(\Omega,\left\{(\alpha, \beta)^{G}: \alpha, \beta \in \Omega\right\}\right)
$$

is a coherent configuration; we say that $\operatorname{Inv}(G)$ is the coherent configuration associated with $G$. A coherent configuration $\mathcal{X}$ is said to be schurian if $\mathcal{X}=\operatorname{Inv}(\operatorname{Aut}(\mathcal{X}))$.

\subsection{Separability}

Let $\mathcal{X}=(\Omega, S)$ and $\mathcal{X}^{\prime}=\left(\Omega^{\prime}, S^{\prime}\right)$ be coherent configurations. A bijection $\varphi: S \rightarrow$ $S^{\prime}, s \mapsto s^{\prime}$, is called an algebraic isomorphism from $\mathcal{X}$ to $\mathcal{X}^{\prime}$ if

$$
c_{r s}^{t}=c_{r^{\prime} s^{\prime}}^{t^{\prime}}, \quad r, s, t \in S .
$$

When $\mathcal{X}=\mathcal{X}^{\prime}$, the set of all such $\varphi$ forms a subgroup of $\operatorname{Sym}(S)$, denoted by $\operatorname{Aut}_{\text {alg }}(\mathcal{X})$.

Each isomorphism $f$ from $\mathcal{X}$ to $\mathcal{X}^{\prime}$ induces an algebraic isomorphism from $\mathcal{X}$ to $\mathcal{X}^{\prime}$, which maps $r \in S$ to $r^{f} \in S^{\prime}$. A coherent configuration $\mathcal{X}$ is said to be separable if every algebraic isomorphism from $\mathcal{X}$ is induced by a suitable bijection (which is an isomorphism of the coherent configurations in question).

The algebraic isomorphism $\varphi$ induces a bijection from $S^{\cup}$ to $\left(S^{\prime}\right)^{\cup}$ : the union $r \cup s \cup \ldots$ of basis relations of $\mathcal{X}$ is mapped to $r^{\prime} \cup s^{\prime} \cup \cdots$. This bijection is also denoted by $\varphi$. It preserves the dot product, i.e., $\varphi(r \cdot s)=\varphi(r) \cdot \varphi(s)$ for all $r, s \in S$. 


\subsection{Coherent closure}

There is a natural partial order $\leq$ on the set of all rainbows on the same set $\Omega$. Namely, given two such rainbows $\mathcal{X}$ and $\mathcal{X}^{\prime}$, we set

$$
\mathcal{X} \leq \mathcal{X}^{\prime} \Leftrightarrow S(\mathcal{X})^{\cup} \subseteq S\left(\mathcal{X}^{\prime}\right)^{\cup}
$$

The minimal and maximal elements with respect to this order are the trivial and discrete coherent configurations, respectively: the basis relations of the former are the reflexive relation $1_{\Omega}$ and (if $|\Omega|>1$ ) its complement in $\Omega \times \Omega$, whereas the basis relations of the latter are singletons.

The functors $\mathcal{X} \rightarrow \operatorname{Aut}(\mathcal{X})$ and $G \rightarrow \operatorname{Inv}(G)$ form a Galois correspondence between the posets of coherent configurations and permutation groups on the same set, i.e.,

$$
\mathcal{Y} \leq \mathcal{X} \Rightarrow \operatorname{Aut}(\mathcal{Y}) \geq \operatorname{Aut}(\mathcal{X}) \quad \text { and } \quad L \leq K \Rightarrow \operatorname{Inv}(L) \geq \operatorname{Inv}(K),
$$

and

$$
\operatorname{Aut}(\operatorname{Inv}(\operatorname{Aut}(\mathcal{X})))=\operatorname{Aut}(\mathcal{X}) \quad \text { and } \quad \operatorname{Inv}(\operatorname{Aut}(\operatorname{Inv}(G)))=\operatorname{Inv}(G) .
$$

The coherent closure $\mathrm{WL}(T)$ of a set $T$ of relations on $\Omega$, is defined to be the smallest coherent configuration on $\Omega$, for which $T$ is a set of relations. The point extension $\mathcal{X}_{\alpha, \beta, \ldots}$ of the rainbow $\mathcal{X}$ with respect to the points $\alpha, \beta, \ldots \in \Omega$ is defined to be $\mathrm{WL}(T)$, where $T$ consists of $S(\mathcal{X})$ and the relations $1_{\alpha}, 1_{\beta}, \ldots$ In other words, $\mathcal{X}_{\alpha, \beta, \ldots}$ is the smallest coherent configuration on $\Omega$ that is larger than or equal to $\mathcal{X}$ and has singletons $\{\alpha\},\{\beta\}, \ldots$ as fibers.

\subsection{Multidimensional intersection numbers}

The theory of multidimensional extensions of coherent configurations has been developed in [4], see also [2, Section 3.5].

Let $m \geq 1$ be an integer. The $m$-extension of a coherent configuration $\mathcal{X}$ on $\Omega$ is defined to be the smallest coherent configuration on $\Omega^{m}$, which contains the Cartesian $m$ power of $\mathcal{X}$ and for which the set $\operatorname{Diag}\left(\Omega^{m}\right)$ is a union of fibers. The intersection numbers of the $m$-extension are called the $m$-dimensional intersection numbers of the configuration $\mathcal{X}$. If $m=1$, then the $m$-extension of $\mathcal{X}$ coincides with $\mathcal{X}$ and the $m$-dimensional intersection numbers of $\mathcal{X}$ are the ordinary intersection numbers.

An algebraic isomorphism $\varphi$ from $\mathcal{X}$ to $\mathcal{X}^{\prime}$ is said to be $m$-dimensional if it can be extended to an algebraic isomorphism from the $m$-extension of $\mathcal{X}$ to that of $\mathcal{X}^{\prime}$, that takes $\operatorname{Diag}\left(\Omega^{m}\right)$ to $\operatorname{Diag}\left(\Omega^{\prime m}\right)$. The separability number $s(\mathcal{X})$ of the coherent configuration $\mathcal{X}$ is defined to be the smallest positive integer $m$ for which every $m$-dimensional algebraic isomorphism from $\mathcal{X}$ is induced by some isomorphism. Thus, the equality $s(\mathcal{X})=m$ expresses the fact that $\mathcal{X}$ is determined up to isomorphism by its tensor of the $m$-dimensional intersection numbers. The following statement was proved in [4, Theorem 4.6(1)].

Lemma 2.1. Let $\mathcal{X}$ be a coherent configuration. Then $s(\mathcal{X}) \leq s\left(\mathcal{X}_{\alpha}\right)+1$ for any point $\alpha$ of $\mathcal{X}$.

\subsection{Pseudocyclic schemes}

Let $\mathcal{X}=(\Omega, S)$ be a coherent configuration. The indistinguishing number of a relation $s \in S(\mathcal{X})$ is defined to be the sum $c(s)$ of the intersection numbers $c_{r r^{*}}^{s}, r \in S$. For each 
pair $(\alpha, \beta) \in s$, we have $c(s)=|c(\alpha, \beta)|$, where

$$
c(\alpha, \beta)=\{\gamma \in \Omega: r(\gamma, \alpha)=r(\gamma, \beta)\} .
$$

The maximum $c(\mathcal{X})$ of the numbers $c(s)$, where $s$ runs over the set of all irreflexive basis relations of $\mathcal{X}$, is called the indistinguishing number of $\mathcal{X}$. It is easily seen that $c=0$ if and only if $n_{s}=1$ for each $s \in S$.

Assume that $\mathcal{X}$ is a scheme. In accordance with [13, Theorem 3.2], $\mathcal{X}$ is pseudocyclic of valency $k$ if the equalities

$$
c(s)+1=k=n_{s}
$$

hold for all irreflexive $s \in S$. The class of pseudocyclic schemes includes all (homogeneous) coherent configurations associated with regular or Frobenius groups.

\subsection{Partly regular coherent configurations}

A coherent configuration $\mathcal{X}$ is said to be partly regular if there exists a point $\alpha \in \Omega$ such that $|\alpha s| \leq 1$ for all $s \in S$; the point $\alpha$ is said to be regular. When all the points of $\mathcal{X}$ are regular, we say that $\mathcal{X}$ is semiregular, and regular if $\mathcal{X}$ is a scheme. Thus, $\mathcal{X}$ is semiregular if and only if $c(\mathcal{X})=0$.

The following statement taken from [2, Theorem 3.3.19] shows, in particular, that the partly regular (respectively, semiregular, regular) coherent configurations are in one-to-one correspondence with those of the form $\operatorname{Inv}(G)$, where $G$ is a permutation group having a faithful orbit (respectively, $G$ is semiregular, regular).

Theorem 2.2. Every partly regular coherent configuration $\mathcal{X}$ is schurian and separable. In particular, $s(\mathcal{X})=1$.

The key point in the proof of Theorem 2.2 is the lemma below [2, Lemma 3.3.20]; it is also used in the proof of Theorem 1.3.

Lemma 2.3. Let $\mathcal{X}$ be a coherent configuration and $\Delta$ a union of fibers of $\mathcal{X}$. Assume that for every $\Gamma \in F$ there exists $s \in S_{\Delta, \Gamma}$ such that $n_{s}=1$. Then

(1) the restriction mapping $\operatorname{Aut}(\mathcal{X}) \rightarrow \operatorname{Aut}\left(\mathcal{X}_{\Delta}\right)$ is a group isomorphism,

(2) $\mathcal{X}$ is schurian and separable whenever $\mathcal{X}_{\Delta}$ is schurian and separable.

\section{Large Hollmann schemes}

\subsection{General properties}

Throughout this section, $d \geq 3$ is an integer, $q=2^{d}$, and $G=\operatorname{PSL}(2, q)$ the permutation group of degree $n=q(q-1) / 2$ from Theorem 1.1(1). The lemma below immediately follows from Theorem 1.2(iii) and Lemma 6.2 proved in [1].

Lemma 3.1. For any point $\alpha$, we have $G_{\alpha}=D_{2(q+1)}$. Moreover,

$$
|\Delta|=q+1, \quad \Delta \in \operatorname{Orb}\left(G_{\alpha}\right), \Delta \neq\{\alpha\} .
$$

To study the large Hollmann scheme $\mathcal{X}=\operatorname{Inv}(G)$, we make use of some results proved in [8]. However, formally, the definition of the scheme given there is different from the 
definition of $\mathcal{X}$. Thus our first goal is to verify that $\mathcal{X}$ is exactly the symmetric pseudocyclic scheme $\mathcal{X}^{\prime}$ of degree $n$ and valency $q+1$ defined in [13] and studied in [8].

First, we note that $\operatorname{Aut}(\mathcal{X})$ is a $\frac{3}{2}$-transitive group of the same degree as $G$; in particular, $\operatorname{Aut}(\mathcal{X})$ is not a subgroup of $\operatorname{A\Gamma L}(1, r)$ for some $r$. Moreover, it is neither 2-transitive, nor Frobenius (because $G \leq \operatorname{Aut}(\mathcal{X})$ ). By Theorem 1.1 and Remark 1.2, this implies that $\operatorname{Aut}(\mathcal{X})=\operatorname{PSL}(2, q)$ or $\operatorname{P\Gamma L}(2, q)$. The latter case is impossible by Lemma 3.1, because $G$ and $\operatorname{Aut}(\mathcal{X})$ have the same subdegrees. Consequently, $G=\operatorname{Aut}(\mathcal{X})$. On the other hand, the scheme $\mathcal{X}^{\prime}$ is also associated with $G$. Therefore a similar argument shows that $\operatorname{Aut}\left(\mathcal{X}^{\prime}\right)=G=\operatorname{Aut}(\mathcal{X})$. Thus,

$$
\mathcal{X}^{\prime}=\operatorname{Inv}\left(\operatorname{Aut}\left(\mathcal{X}^{\prime}\right)\right)=\operatorname{Inv}(\operatorname{Aut}(\mathcal{X}))=\mathcal{X} .
$$

Proposition 3.2. The large Hollmann scheme $\mathcal{X}$ is symmetric and pseudocyclic of degree $q(q-1) / 2$, rank $q / 2$, and valency $q+1$. Moreover,

$$
\operatorname{Aut}(\mathcal{X})=G \quad \text { and } \operatorname{Aut}\left(\mathcal{X}_{\alpha}\right)=G_{\alpha}=D_{2(q+1)} \text { for all } \alpha
$$

Proof. By the remark before the proposition, we need to verify the second equality in (3.3) only. By [2, Proposition 3.3.3(1)], we have $\operatorname{Aut}\left(\mathcal{X}_{\alpha}\right)=\operatorname{Aut}(\mathcal{X})_{\alpha}$. Thus the required statement immediately follows from the first equality in (3.3) and Lemma 3.1.

Equality (3.2) allows us to use formulas for the intersection numbers of the scheme $\mathcal{X}^{\prime}$, given in [8, Theorem 2.2]. Namely, let $S=S(\mathcal{X})$ and

$$
\mathbf{T}_{0}=\left\{x \in \mathbb{F}_{2^{d}}: \operatorname{Tr}(x)=0\right\},
$$

where $\operatorname{Tr}(x)$ is the trace of $x$ over the prime subfield of the field $\mathbb{F}_{2^{d}}$. Then there is a bijection $\mathbf{T}_{0} \rightarrow S, x \mapsto s_{x}$, such that $s_{0}$ is reflexive and

$$
c_{s_{x}, s_{y}}^{s_{z}}=1 \quad \Leftrightarrow \quad \operatorname{Tr}(x z)=0 \text { and } x+y+z=0 .
$$

As is easily seen, $\mathbf{T}_{0}$ is a linear space of dimension $d-1$ over $\mathbb{F}_{2}$.

\subsection{One point extension}

Let us analyze the extension $\mathcal{X}_{\alpha}$ of the large Hollmann scheme $\mathcal{X}$ with respect to a point $\alpha$. Since the scheme $\mathcal{X}$ is schurian, each fiber of the coherent configuration $\mathcal{X}_{\alpha}$ is of the form $\Delta=\alpha s$ for some $s \in S$ [2, Theorem 3.3.7]. When $s=s_{x}$ for some $x \in \mathbf{T}_{0}$, the fiber $\Delta$ is denoted by $\Delta_{x}$. Thus,

$$
F\left(\mathcal{X}_{\alpha}\right)=\left\{\Delta_{x}: x \in \mathbf{T}_{0}\right\}
$$

Theorem 3.3. Let $x$ and $y$ be nonzero elements of $\mathbf{T}_{0}$. Then the set $S\left(\mathcal{X}_{\alpha}\right)_{\Delta_{x}, \Delta_{y}}$ contains a matching.

Proof. For $d=3$, the statement has been checked with the help of the computer package $\mathrm{COCO} 2 \mathrm{P}[9]$. Assume that $d \geq 4$. We need auxiliary lemmas.

Lemma 3.4. Theorem 3.3 holds whenever $\operatorname{Tr}(x y)=0$. 
Proof. Let $z=x+y$. Then obviously $z \in \mathbf{T}_{0}$. Moreover

$$
\operatorname{Tr}(x z)=\operatorname{Tr}\left(x^{2}+x y\right)=\operatorname{Tr}\left(x^{2}\right)+\operatorname{Tr}(x y)=0 .
$$

By formula (3.4), this implies that $c_{s_{x}, s_{y}}^{s_{z}}=1$. If $z=0$, then $x=y$ and $1_{\Delta_{x}}$ is a desired matching. Assume that $z$ is nonzero. Then $n_{s_{x}}=n_{s_{z}}$, because $\mathcal{X}$ is a pseudocyclic scheme (Proposition 3.2). Since $\mathcal{X}$ is also symmetric, we have

$$
c_{s_{y}, s_{z}}^{s_{x}}=\frac{n_{s_{z}}}{n_{s_{x}}} c_{s_{x}, s_{y}}^{s_{z}}=1,
$$

see (2.1). Therefore if $r=s_{z} \cap\left(\alpha s_{x} \times \alpha s_{y}\right)$, then $|\beta r|=1$ for all $\beta \in \Delta_{x}$, and $\left|\beta r^{*}\right|=1$ for all $\beta \in \Delta_{y}$ (here we use the fact that $\left|\Delta_{x}\right|=\left|\Delta_{y}\right|$ ). Since $r$ is a relation of $\mathcal{X}_{\alpha}$ [2, Lemma 3.3.5], this implies that $r$ belongs to $S\left(\mathcal{X}_{\alpha}\right)_{\Delta_{x}, \Delta_{y}}$. Thus, $r$ is a required matching.

Let us define a graph $\mathfrak{X}$ with nonzero elements of $\mathbf{T}_{0}$ as the vertices and in which two distinct vertices $x$ and $y$ are adjacent if and only if $\operatorname{Tr}(x y)=0$. One can see that $\mathfrak{X}$ is an undirected graph with exactly $\left|\mathbf{T}_{0}\right|-1$ vertices.

Lemma 3.5. The graph $\mathfrak{X}$ is connected.

Proof. Given $x \in \mathbf{T}_{0}$, we define the linear mapping

$$
f_{x}: \mathbf{T}_{0} \rightarrow \mathbb{F}_{2}, y \mapsto \operatorname{Tr}(x y) .
$$

Now let $x \neq y$ be two vertices of the graph $\mathfrak{X}$. If $\operatorname{Tr}(x y)=0$, then $x$ and $y$ are connected by an edge. Let $\operatorname{Tr}(x y) \neq 0$. Then both $f_{x}$ and $f_{y}$ are nonzero linear mappings, and $\operatorname{ker}\left(f_{x}\right)$ and $\operatorname{ker}\left(f_{y}\right)$ are subspaces of $\mathbf{T}_{0}$ of codimension one. Therefore, $\operatorname{ker}\left(f_{x}\right) \cap \operatorname{ker}\left(f_{y}\right)$ is a subspace of $\mathbf{T}_{0}$ of codimension at most two. Consequently,

$$
\operatorname{dim}\left(\operatorname{ker}\left(f_{x}\right) \cap \operatorname{ker}\left(f_{y}\right)\right) \geq \operatorname{dim}\left(\mathbf{T}_{0}\right)-2=d-3 \geq 1 .
$$

It follows that $\operatorname{ker}\left(f_{x}\right) \cap \operatorname{ker}\left(f_{y}\right)$ contains at least one nonzero vector, say $z$. Then $\operatorname{Tr}(x z)=$ $\operatorname{Tr}(y z)=0$. Since $\operatorname{Tr}(x y) \neq 0$, this implies that $z \neq x, y$. Thus the vertices $x, y$ are at distance two in the graph $\mathfrak{X}$, in particular, $\mathfrak{X}$ is connected.

Let us return to the proof of Theorem 3.3. By Lemma 3.5, the vertices $x$ and $y$ of the graph $\mathfrak{X}$ are connected by a path

$$
x=x_{0}, x_{1}, \ldots, x_{k}=y,
$$

where $k \geq 1$. For $i=0, \ldots, k-1$, the vertices $x_{i}$ and $x_{i+1}$ are adjacent and hence $\operatorname{Tr}\left(x_{i} x_{i+1}\right)=0$. Denote by $s_{i}$ the matching in $S\left(\mathcal{X}_{\alpha}\right)_{\Delta_{x_{i}}, \Delta_{x_{i+1}}}$ the existence of which is guaranteed by Lemma 3.4. Then the dot product

$$
s=s_{0} \cdot s_{1} \cdots s_{k-1}
$$

is a desired matching belonging to $S\left(\mathcal{X}_{\alpha}\right)_{\Delta_{x}, \Delta_{y}}$.

Corollary 3.6. Let $\Delta=\Delta_{x}$ for nonzero $x \in \mathbf{T}_{0}$. Then the coherent configuration $\mathcal{Y}=$ $\left(\mathcal{X}_{\alpha}\right)_{\Delta}$ is schurian and separable. Moreover, the extension of $\mathcal{Y}$ with respect to at least one point is partly regular. 
Proof. By Proposition 3.2, we have $\operatorname{Aut}\left(\mathcal{X}_{\alpha}\right)=D_{2(q+1)}$. Furthermore, the hypothesis of Lemma 2.3 is satisfied for $\mathcal{X}=\mathcal{X}_{\alpha}$ by Theorem 3.3. Thus by statement (1) of that lemma, we have

$$
H:=\operatorname{Aut}(\mathcal{Y}) \cong \operatorname{Aut}\left(\mathcal{X}_{\alpha}\right)=D_{2(q+1)} .
$$

On the other hand, $|\Delta|=q+1$ by formula (3.1). Consequently, the group $H$ contains a normal regular cyclic subgroup $C$ of order $q+1$. In terms of [2, Section 4.4], this means that $\mathcal{Y}$ is isomorphic to a normal circulant scheme. The radical of such a scheme, being a subgroup of the group $C$, is of order at most 2 ; this follows from the implication $(1) \Leftrightarrow(3)$ in [5, Theorem 6.1]. Since the number $|C|=q+1=2^{d}+1$ is odd, the radical is trivial. Thus, the scheme $\mathcal{Y}$ is schurian by [2, Corollary 4.4.3], and every its extension with respect to at least one point is partly regular by [2, Theorem 4.4.7].

It remains to verify that $\mathcal{Y}$ is separable. Since $\mathcal{Y}$ is schurian by above, we have $\mathcal{Y}=$ $\operatorname{Inv}(\operatorname{Aut}(\mathcal{Y}))=\operatorname{Inv}(H)$. By virtue of (3.5), this means that $\mathcal{Y}$ is the coherent configuration associated with $D_{2(q+1)}$. Thus, the required statement follows from [2, Exercise 2.7.33].

\subsection{Proof of Theorem 1.3}

By Lemma 2.1, it suffices to verify that a one point extension of a large Hollmann scheme is separable. But this immediately follows from Theorem 3.7 below.

Theorem 3.7. Let $q=2^{d}$ where $d \geq 3$. Then the extension of the large Hollmann scheme of degree $q(q-1) / 2$ with respect to at least one point is schurian and separable.

Proof. Let $\mathcal{X}^{\prime}$ be the extension of the large Hollmann scheme $\mathcal{X}$ with respect to $m \geq 1$ points $\alpha=\alpha_{1}, \alpha_{2}, \ldots, \alpha_{m}$. Let $x \in \mathbf{T}_{0}$ be nonzero and $\Delta=\Delta_{x}$. Then the hypothesis of Lemma 2.3 is satisfied for $\mathcal{X}=\mathcal{X}^{\prime}$. Indeed, each $\Gamma \in F\left(\mathcal{X}^{\prime}\right)$ other than $\{\alpha\}$ is contained in $\Delta_{y}$ for some nonzero $y \in \mathbf{T}_{0}$. By Theorem 3.3, there is a matching $s^{\prime} \in S\left(\mathcal{X}_{\alpha}\right)_{\Delta_{x}, \Delta_{y}}$, and as the required relation $s$ one can take $s^{\prime} \cap(\Delta \times \Gamma)$.

By Lemma 2.3(2), the coherent configuration $\mathcal{X}^{\prime}$ is schurian and separable whenever so is $\mathcal{X}_{\Delta}^{\prime}$. If $m=1$, then $\mathcal{X}^{\prime}=\mathcal{X}_{\alpha}$ and we are done by Corollary 3.6. Let $m>1$. We claim that there exist $\beta_{2}, \ldots, \beta_{m} \in \Delta$ such that

$$
\mathcal{X}^{\prime}=\mathcal{X}_{\alpha, \beta_{2}, \ldots, \beta_{m}}
$$

Indeed, without loss of generality we may assume that none of the $\alpha_{i}, i>1$, equals $\alpha$. By Theorem 3.3, there is a matching $s_{i} \in S\left(\mathcal{X}_{\alpha}\right)_{\Delta_{i}, \Delta}$, where $\Delta_{i}$ is the fiber of $\mathcal{X}_{\alpha}$, containing $\alpha_{i}$. Then $\alpha_{i} s_{i}=\left\{\beta_{i}\right\}$ for some $\beta_{i} \in \Delta$. It follows that for any extension of $\mathcal{X}_{\alpha}$, each or none of the two singletons $\left\{\alpha_{i}\right\}$ and $\left\{\beta_{i}\right\}$ is a fiber of this extension, see [2, Corollary 3.3.6]. Thus,

$$
\mathcal{X}^{\prime}=\mathcal{X}_{\alpha, \alpha_{2}, \ldots, \alpha_{m}}=\mathcal{X}_{\alpha, \beta_{2}, \ldots, \beta_{m}},
$$

which completes the proof of the claim.

Now from formula (3.6) and the fact that $\beta_{i} \in \Delta$ for all $i$, it easily follows that

$$
\begin{aligned}
\mathcal{X}_{\Delta}^{\prime} & =\left(\mathcal{X}_{\alpha, \alpha_{2}, \ldots, \alpha_{m}}\right)_{\Delta}=\left(\mathcal{X}_{\alpha, \beta_{2}, \ldots, \beta_{m}}\right)_{\Delta} \\
& =\left(\left(\mathcal{X}_{\alpha, \beta_{2}, \ldots, \beta_{m}}\right)_{\Delta}\right)_{\beta_{2}, \ldots, \beta_{m}} \geq\left(\left(\mathcal{X}_{\alpha}\right)_{\Delta}\right)_{\beta_{2}, \ldots, \beta_{m}} .
\end{aligned}
$$

The coherent configuration on the right-hand side of this relation is partly regular by Corollary 3.6. Therefore, the coherent configuration $\mathcal{X}_{\Delta}^{\prime}$ is also partly regular. By Theorem 2.2, this implies that $\mathcal{X}_{\Delta}^{\prime}$ is schurian and separable, as required. 


\section{A lower bound for indistinguishing number}

The main result of this section (Theorem 4.1 below) establishes a lower bound for the indistinguishing number of a coherent configuration which is not partly regular, cf. [17, Theorem 3.1]. This bound gives a sufficient condition for a coherent configuration to be partly regular, and is used to prove Theorems 1.4 and 1.5 in the next section.

Theorem 4.1. Let $\mathcal{X}$ be a coherent configuration of degree $n, k$ the maximal cardinality of a fiber of $\mathcal{X}$, and $c=c(\mathcal{X})$. If $\mathcal{X}$ is not partly regular, then

$$
(2 k-1) c \geq n
$$

Proof. Let $\mathcal{X}=(\Omega, S)$. In the sequel, $\Delta \in F(\mathcal{X})$ and $|\Delta|=k$. The fiber $\Delta$ contains at least two points, for otherwise $k=1$ and $\mathcal{X}$ is the discrete and hence partly regular configuration in contrast to the hypothesis of the theorem. Set

$$
\Delta_{\alpha}=\left\{\delta \in \Delta: n_{r(\alpha, \delta)}=1\right\} \quad \text { and } \quad \Omega_{1}=\left\{\alpha \in \Omega: \Delta_{\alpha}=\Delta\right\} .
$$

Lemma 4.2. $\Omega_{1}$ is a (possibly empty) union of fibers of cardinality $k$. Moreover, the coherent configuration $\mathcal{X}_{\Omega_{1}}$ is semiregular.

Proof. Let $\Gamma$ be a fiber containing a point of $\Omega_{1}$. The set $S_{\Gamma, \Delta}$ consists of $s=r(\gamma, \delta)$, where $\gamma \in \Gamma \cap \Omega_{1}$ and $\delta$ runs over $\Delta$. Consequently, $n_{s}=1$ for all $s \in S_{\Gamma, \Delta}$. Therefore, $\Gamma \subseteq \Omega_{1}$. Thus, $\Omega_{1}$ is a union of fibers of $\mathcal{X}$. Furthermore, given $s \in S_{\Gamma, \Delta}$ we have

$$
k \geq|\Gamma|=n_{s}|\Gamma|=n_{s^{*}}|\Delta| \geq|\Delta|=k .
$$

This proves the first statement. To prove the second one, let $\Delta^{\prime}$ and $\Delta^{\prime \prime}$ be fibers contained in $\Omega_{1}$. By the definition of $\Omega_{1}$ and the first statement, any relations $s^{\prime} \in S_{\Delta^{\prime}, \Delta}$ and $s^{\prime \prime} \in$ $S_{\Delta, \Delta^{\prime \prime}}$ are matchings. Therefore, $s^{\prime} \cdot s^{\prime \prime}$ is a matching contained in $S_{\Delta^{\prime}, \Delta^{\prime \prime}}$. Thus, $S_{\Omega_{1}}$ consists of matchings and we are done.

By Lemma 4.2 and the hypothesis of the theorem, the complement $\Omega^{\prime}$ of the set $\Omega_{1}$ in $\Omega$ contains at least two distinct points.

Lemma 4.3. For each $\gamma \in \Omega^{\prime}$,

$$
\sum_{s \in S_{\gamma}} n_{s} \geq \frac{k}{2}
$$

where $S_{\gamma}=\left\{r(\gamma, \delta): \delta \in \Delta\right.$ and $\left.n_{r(\gamma, \delta)}>1\right\}$.

Proof. We have

$$
\sum_{s \in S_{\gamma}} n_{s}=\sum_{s \in S_{\gamma}}|\gamma s|=|\Delta|-\left|\Delta_{\gamma}\right| .
$$

Since $|\Delta|=k$, this proves the required inequality if $\Delta_{\gamma}=\varnothing$. Let $\delta \in \Delta_{\gamma}$. It is easily seen that $r(\delta, \lambda)=r(\delta, \gamma) \cdot r(\gamma, \lambda)$ is a matching of $\mathcal{X}_{\Delta}$ for each $\lambda \in \Delta_{\gamma}$. Therefore,

$$
\Delta_{\gamma} \subseteq\left\{\lambda \in \Delta: n_{r(\delta, \lambda)}=1\right\}
$$

On the other hand, denote by $e$ the union of all matchings of the scheme $\mathcal{X}_{\Delta}$. Then $e$ is a relation of this scheme. Moreover, $e$ is an equivalence relation on $\Delta$ (see [2, Theorem 2.1.25(4)]) and the set on the right-hand side of (4.3) is a class of $e$. In view of [2, 
Corollary 2.1.23], the cardinality $a$ of this class divides $|\Delta|=k$. Furthermore, $a \neq k$, for otherwise, $\Delta_{\gamma}=\Delta$ and then $\gamma \in \Omega_{1}$, a contradiction. Thus,

$$
\left|\Delta_{\gamma}\right| \leq a \leq \frac{k}{2}
$$

and the required statement follows from (4.2).

Lemma 4.4. Let $\varepsilon=\frac{2(k-1)}{2 k-1}$. Assume that $\left|\Omega^{\prime}\right| \geq \varepsilon n$. Then inequality (4.1) holds.

Proof. Denote by $N$ the cardinality of the set

$$
\left\{(\alpha, \beta, \gamma) \in \Delta \times \Delta \times \Omega^{\prime}: \alpha \neq \beta, \gamma \in c(\alpha, \beta)\right\},
$$

where $c(\alpha, \beta)$ is as in formula (2.3). The number of $(\alpha, \beta) \in \Delta \times \Delta$ with $\alpha \neq \beta$ is equal to $k(k-1)$. Therefore there exists at least one such pair for which

$$
|c(\alpha, \beta)| \geq \frac{N}{k(k-1)} .
$$

On the other hand, let $\gamma \in \Omega^{\prime}$, and let $S_{\gamma}$ be as in Lemma 4.3. Then $n_{s} \geq 2$ for all $s \in S_{\gamma}$. For every such $s$ there are exactly $n_{s}\left(n_{s}-1\right)$ triples $(\alpha, \beta, \gamma)$ with distinct $\alpha, \beta \in \gamma s$, and all these triples belong to the set (4.4). By Lemma 4.3 this implies that

$$
N=\sum_{\gamma \in \Omega^{\prime}} \sum_{s \in S_{\Gamma, \Delta}} n_{s}\left(n_{s}-1\right) \geq \sum_{\gamma \in \Omega^{\prime}} \sum_{s \in S_{\gamma}} n_{s} \geq \sum_{\gamma \in \Omega^{\prime}} \frac{k}{2}=\frac{\left|\Omega^{\prime}\right| k}{2},
$$

where $\Gamma$ is the fiber containing $\gamma$. By formula (4.5) and the lemma assumption, we obtain

$$
c \geq|c(\alpha, \beta)| \geq \frac{N}{k(k-1)} \geq \frac{\left|\Omega^{\prime}\right|}{2(k-1)} \geq \frac{2(k-1) n}{2 k-1} \cdot \frac{1}{2(k-1)}=\frac{n}{2 k-1},
$$

as required.

By Lemma 4.4, we may assume that $\left|\Omega^{\prime}\right|<\varepsilon n$. The coherent configuration $\mathcal{X}$ is not partly regular. Therefore no point $\delta \in \Omega_{1}$ is regular and there exist distinct $\alpha, \beta \in \Omega^{\prime}$ such that $\delta \in c(\alpha, \beta)$. Since the coherent configuration $\mathcal{X}_{\Omega_{1}}$ is semiregular (Lemma 4.2), the relation $s=r(\delta, \lambda)$ is a matching for all $\lambda \in \Omega_{1}$. It follows that

$$
r(\alpha, \lambda)=r(\alpha, \delta) \cdot s=r(\beta, \delta) \cdot s=r(\beta, \lambda) .
$$

Consequently, $\Omega_{1} \subseteq c(\alpha, \beta)$. This implies that

$$
c \geq|c(\alpha, \beta)| \geq\left|\Omega_{1}\right|=n-\varepsilon n>n\left(1-\frac{2(k-1)}{2 k-1}\right)=\frac{n}{2 k-1}
$$

which completes the proof of Theorem 4.1.

Corollary 4.5. Let $\mathcal{X}$ be a coherent configuration of degree $n, c=c(\mathcal{X})$, and $t$ an irreflexive basis relation of $\mathcal{X}$. Assume that $\left(2 m_{t}-1\right) c<n$, where

$$
m_{t}=\max _{r, s \in S} c_{r s}^{t} \text {. }
$$

Then the extension of $\mathcal{X}$ with respect to any two points forming a pair from $t$ is partly regular. 
Proof. Let $\mathcal{X}^{\prime}$ be the extension of $\mathcal{X}$ with respect to the points $\alpha, \beta$ such that $(\alpha, \beta) \in t$. Then each fiber $\Delta$ of $\mathcal{X}^{\prime}$ different from both $\{\alpha\}$ and $\{\beta\}$ is contained in the set $\alpha r \cap \beta s^{*}$ for appropriate $r, s \in S$. It follows that

$$
|\Delta| \leq\left|\alpha r \cap \beta s^{*}\right|=c_{r s}^{t} \leq m_{t}
$$

Thus the maximal cardinality $k^{\prime}$ of a fiber of $\mathcal{X}^{\prime}$ is less than or equal to $m_{t}$. Since obviously $c^{\prime}=c\left(\mathcal{X}^{\prime}\right)$ is less than or equal to $c$, the condition of the corollary implies that

$$
\left(2 k^{\prime}-1\right) c^{\prime} \leq\left(2 m_{t}-1\right) c<n .
$$

Thus $\mathcal{X}^{\prime}$ is partly regular by Theorem 4.1 .

\section{Small Hollmann and Passman schemes}

\subsection{Algebraic fusion}

Let $\mathcal{X}=(\Omega, S)$ be a coherent configuration, and let $\Phi$ be a group of algebraic automorphisms of $\mathcal{X}$. For each $s \in S$, set

$$
s^{\Phi}=\bigcup_{\varphi \in \Phi} \varphi(s) .
$$

Clearly, $\left(1_{\Omega}\right)^{\Phi}=1_{\Omega}$. Moreover the set $S^{\Phi}=\left\{s^{\Phi}: s \in S\right\}$ forms a partition of the Cartesian square $\Omega^{2}$. According to [2, Lemma 2.3.26], the pair

$$
\mathcal{X}^{\Phi}=\left(\Omega, S^{\Phi}\right)
$$

is a coherent configuration called the algebraic fusion of $\mathcal{X}$ with respect to $\Phi$. In the following lemma, we establish a simple upper bound for the intersection numbers of an algebraic fusion.

Lemma 5.1. In the above notation, let $r, s, t \in S$ and $m_{t}$ be as in (4.6). Then

$$
c_{r^{\Phi} s^{\Phi}}^{t^{\Phi}} \leq m_{t}|\Phi|^{2}
$$

Proof. We have $c_{r^{\Phi} s^{\Phi}}^{t^{\Phi}}=\sum_{\varphi, \psi \in \Phi} c_{\varphi(r) \psi(s)}^{t} \leq m_{t}|\Phi|^{2}$.

\subsection{Proof of Theorem 1.4}

Let $\mathcal{X}$ be a small Hollmann scheme. Then the degree of $\mathcal{X}$ is equal to $n=q(q-1) / 2$, where $q=2^{d}$ for a prime $d \geq 3$. Moreover, $\mathcal{X}$ is associated with the permutation group $G=\operatorname{P\Gamma L}(2, q)$ of degree $n$ from Theorem 1.1(1). As in Subsection 3.1, one can see that $\mathcal{X}$ coincides with symmetric pseudocyclic scheme $\mathcal{X}^{\prime}$ of degree $n$ and valency $d(q+1)$, associated with the group $\operatorname{P\Gamma L}(2, q)$ and studied in [8]. In particular, $\mathcal{X}$ is obtained from the large Hollmann scheme of degree $n$ by merging the basis relations via the Frobenius map $x \mapsto x^{2}, x \in \mathbb{F}_{q}$. In other words, $\mathcal{X}$ is the algebraic fusion of the large Hollmann scheme of degree $n$ with respect the induced action of $\operatorname{Aut}\left(\mathbb{F}_{q}\right)$ on its basis relations.

Proposition 5.2. Let $\mathcal{X}$ and $\mathcal{X}_{q}$ be the small and large Hollmann schemes of degree $q(q-$ 1) $/ 2$, respectively. Then 
(1) $\mathcal{X}=\mathcal{X}_{q}^{\Phi}$, where $\Phi \leq \operatorname{Aut}_{\text {alg }}\left(\mathcal{X}_{q}\right)$ is a group of order $d$,

(2) $\mathcal{X}$ is a pseudocyclic scheme of valency $d(q+1)$,

(3) for each irreflexive $t \in S(\mathcal{X})$, we have $m_{t} \leq 4 d^{2}$, where $m_{t}$ is as in (4.6).

Proof. Statements (1) and (2) follow from the above discussion. Next, from the formulas for the intersection numbers of the scheme $\mathcal{X}_{q}$, given in [8, Theorem 2.2], it follows that $c_{x y}^{z} \leq 4$ for all irreflexive $x, y, z \in S\left(\mathcal{X}_{q}\right)$. In particular,

$$
m_{z} \leq 4
$$

On the other hand, by statement (1), each irreflexive $t \in S(\mathcal{X})$ is of the form $z^{\Phi}$ for some irreflexive $z$. Thus by Lemma 5.1,

$$
m_{t}=\max _{x, y \in S\left(\mathcal{X}_{q}\right)} c_{x^{\Phi} y^{\Phi}}^{z^{\Phi}} \leq m_{z}|\Phi|^{2} \leq 4 d^{2},
$$

which proves statement (3).

Let us prove Theorem 1.4. If $d=3$, then a straightforward calculation shows that $\mathcal{X}$ is trivial scheme and hence $c(\mathcal{X})=1$. Let $d>3$. By Lemma 2.1 and Theorem 2.2, it suffices to verify that the extension of $\mathcal{X}$ with respect to at least two points is partly regular.

Theorem 5.3. Let $q=2^{d}$, where $d>3$ is a prime and $d \neq 7,11,13$. Then every extension of the small Hollmann scheme of degree $q(q-1) / 2$ with respect to at least two points is partly regular.

Proof. Let $\mathcal{X}$ be the small Hollmann scheme of degree $n=q(q-1) / 2$. By Proposition 5.2(2), the number $c=c(\mathcal{X})$ is equal to $d\left(2^{d}+1\right)-1$. By statement (3) of the same proposition, $m_{t} \leq 4 d^{2}$ for any irreflexive $t \in S(\mathcal{X})$. Now if $d>16$, then

$$
\left(2 m_{t}-1\right) c<\left(8 d^{2}-1\right)\left(d\left(2^{d}+1\right)-1\right)<2^{d-1}\left(2^{d}-1\right)=n .
$$

By Corollary 4.5, this proves the required statement for all (prime) $d>13$. In the remaining case, $d=5$, the required statement has been checked with the help of the computer package COCO2P [9].

\subsection{Proof of Theorem 1.5}

Let $q$ be an odd prime power. The permutation group $G \leq \operatorname{AGL}(2, q)$ defined in Theorem 1.1(2) has a Frobenius subgroup $H$ consisting of the permutations

$$
\left(\begin{array}{l}
x \\
y
\end{array}\right) \rightarrow\left(\begin{array}{cc}
a & 0 \\
0 & a^{-1}
\end{array}\right)\left(\begin{array}{l}
x \\
y
\end{array}\right)+\left(\begin{array}{l}
b \\
c
\end{array}\right), \quad x, y \in \mathbb{F}_{q},
$$

where $a, b, c \in \mathbb{F}_{q}$ and $a \neq 0$. The group $D \leq \mathrm{GL}(2, q)$ consisting of the eight matrices

$$
\left(\begin{array}{cc} 
\pm 1 & 0 \\
0 & \pm 1
\end{array}\right) \text { and }\left(\begin{array}{cc}
0 & \pm 1 \\
\pm 1 & 0
\end{array}\right)
$$

is contained in $G$, normalizes $H$, and, moreover, $G=H \rtimes D$. In particular, $D$ acts on the basis relations of the Frobenius scheme $\mathcal{Y}=\operatorname{Inv}(H)$ as a group $\Phi$ of algebraic automorphisms. 
Proposition 5.4. Let $\mathcal{X}=\operatorname{Inv}(G)$ be the Passman scheme of degree $q^{2}$. Then

(1) $\mathcal{X}=\mathcal{Y}^{\Phi}$, where $\Phi \leq \operatorname{Aut}_{\text {alg }}(\mathcal{Y})$ is a group of order 2 ,

(2) $\mathcal{X}$ is a pseudocyclic scheme of valency $2(q-1)$,

(3) there is an irreflexive $t \in S(\mathcal{X})$ such that $m_{t} \leq 8$, where $m_{t}$ is as in (4.6).

Proof. Statements (1) and (2) follow from [13, Section 4.3]. To prove statement (3), let $u$ be the basis relation of $\mathcal{Y}$, containing the pair $(\alpha, \beta)$, where

$$
\alpha=(0,0) \text { and } \beta=(1,1) \text {. }
$$

It suffices to verify that $m_{u} \leq 2$; indeed, then $t=u^{\Phi}$ is the required relation by statement (1) and Lemma 5.1.

We need to verify that $c_{r s}^{u} \leq 2$ for all $r, s \in S(\mathcal{Y})$. Without loss of generality, we may assume that $r$ and $s$ are such that $c_{r s}^{u} \neq 0$. Then there is $\gamma \in \alpha r \cap \beta s^{*}$. It follows that $\alpha r=\gamma^{H_{\alpha}}, \beta s^{*}=\gamma^{H_{\beta}}$, and

$$
c_{r s}^{u}=\left|\gamma^{H_{\alpha}} \cap \gamma^{H_{\beta}}\right| .
$$

Let us calculate the number on the right-hand side. Using the explicit form (5.1) of the elements of $H$, one can easily find that the groups $H_{\alpha}$ and $H_{\beta}$ consist of permutations the parameters $a, b, c$ of which satisfy the relations

$$
b=c=0 \quad \text { and } \quad a+b=a^{-1}+c=1,
$$

respectively. Consequently, assuming $\gamma=(x, y)$, we have

$\gamma^{H_{\alpha}}=\left\{\left(a x, \frac{y}{a}\right): a \in \mathbb{F}_{q}^{*}\right\} \quad$ and $\quad \gamma^{H_{\beta}}=\left\{\left(a^{\prime} x+1-a^{\prime}, \frac{y}{a^{\prime}}+1-\frac{1}{a^{\prime}}\right): a^{\prime} \in \mathbb{F}_{q}^{*}\right\}$.

In view of (5.2), the intersection number $c_{r s}^{u}$ is equal to the number of elements $a \in \mathbb{F}_{q}^{*}$ such that

$$
a x=a^{\prime} x+1-a^{\prime} \quad \text { and } \quad \frac{y}{a}=\frac{y}{a^{\prime}}+1-\frac{1}{a^{\prime}} .
$$

If exactly one of $x, y$ equals 0 , then these equations are satisfied for $a=1=a^{\prime}$ only, and $c_{r s}^{u}=1$. Assume that $x \neq 0 \neq y$. Then

$$
a=a^{\prime}\left(1-\frac{1}{x}\right)+\frac{1}{x} \text { and } \quad \frac{1}{a}=\frac{1}{a^{\prime}}\left(1-\frac{1}{y}\right)+\frac{1}{y}
$$

and hence

$$
1=\left(1-\frac{1}{x}\right)\left(1-\frac{1}{y}\right)+\frac{a^{\prime}}{y}\left(1-\frac{1}{x}\right)+\frac{1}{a^{\prime} x}\left(1-\frac{1}{y}\right)+\frac{1}{x y} .
$$

This gives a quadratic equation with unknown $a^{\prime}$, and at most two possible values for $a^{\prime}$. Thus, $c_{r s}^{u} \leq 2$.

Let us prove Theorem 1.5. By Lemma 2.1 and Theorem 2.2, it suffices to verify that the extension of $\mathcal{X}$ with respect to at least two points is partly regular. 
Theorem 5.5. Let $q$ be an odd prime power. Then every extension of the Passman scheme of degree $q^{2}$ with respect to at least two points is partly regular.

Proof. Let $\mathcal{X}$ be the Passman scheme of degree $n=q^{2}$. By Proposition 5.4(2), the number $c=c(\mathcal{X})$ is equal to $2(q-1)-1$. Let $t$ be the basis relation of $\mathcal{X}$, defined in Proposition 5.4(3). Then $m_{t} \leq 8$. Now if $q \geq 29$, then

$$
\left(2 m_{t}-1\right) c \leq 15(2 q-3)<q^{2}=n .
$$

By Corollary 4.5, this proves the required statement for all $q \geq 29$. In the remaining cases, the required statement has been checked with the help of the computer package $\mathrm{COCO} 2 \mathrm{P}[9]$.

\section{Concluding remarks and open problems}

\subsection{Pseudocyclic schemes}

A scheme is said to be $k$-equivalenced (and just equivalenced if $k$ is irrelevant) if all irreflexive basis relations of it have valency $k$. It is known that every $k$-equivalenced scheme is pseudocyclic for $1 \leq k \leq 4$; this follows from results obtained in [12,14,15] and [13, Theorem 3.1].

By Theorem 1.1, if $\mathcal{X}$ is a schurian equivalenced scheme of sufficiently large degree, which is not trivial or Frobenius, then either $\mathcal{X}$ is exceptional, i.e., the Hollmann or Passman scheme, or the inclusion $\operatorname{Aut}(\mathcal{X}) \leq \operatorname{A\Gamma L}(1, q)$ holds for some $q$. In all cases except for the last one, $\mathcal{X}$ is pseudocyclic, see Propositions 3.2, 5.2(2), and 5.4(2), and [13, Theorem 3.1]. In the latter case, the group $\mathrm{A} \Gamma \mathrm{L}(1, q)$ can contain $\frac{3}{2}$-transitive subgroups which are not 2 equivalent to Frobenius groups (such a subgroup is always primitive). We do not know whether the scheme of at least one of these subgroups is not pseudocyclic.

\subsection{Separability number}

Finding the exact values of $s(\mathcal{X})$ for an exceptional scheme $\mathcal{X}$ is still an open problem. $\mathrm{A}$ direct calculation shows that these schemes are separable for small $q$.

\subsection{Superschurian schemes}

The following concept was first formulated many years ago in discussions of the third author with Sergei Evdokimov. A scheme $\mathcal{X}$ is said to be superschurian if the extension of $\mathcal{X}$ with respect to every set of points is schurian. In particular, all superschurian schemes are schurian. In fact, only a few families of superschurian schemes are known; these include partly regular schemes, cyclotomic schemes over finite fields, normal circulant schemes, and some TI-schemes [3, 5, 13]. Theorem 3.7 implies that any large Hollmann scheme is superschurian. We do not know whether other exceptional schemes are superschurian.

\subsection{Base number}

The base number $b(\mathcal{X})$ of a coherent configuration $\mathcal{X}$ is defined to be the smallest number of points such that the extension of $\mathcal{X}$ with respect to them is the discrete configuration, see [2, Section 3.3.2]. In general, the base number of the group $\operatorname{Aut}(\mathcal{X})$ is less than or equal to $b(\mathcal{X})$. The equality is attained, for example, if $\mathcal{X}$ is a partly regular coherent configuration. By virtue of this observation, Theorems 3.7, 5.3, and 5.5 imply that except, 
possibly, for several small Hollmann schemes the equality holds also for all exceptional pseudocyclic schemes.

In fact, the base number of an exceptional scheme of enough large degree is bounded by 3 . This fact can be used to construct a polynomial-time algorithm recognizing whether or not a given scheme is exceptional. Taking the above discussion into account, this reduces the recognition problem for the class of schurian equivalenced schemes (see [18, p.281]) to the non-Frobenius schemes $\mathcal{X}$ for which $\operatorname{Aut}(\mathcal{X}) \leq \mathrm{A \Gamma L}(1, q)$.

\subsection{Bound in Theorem 4.1}

Denote by $f(n)$ the maximum of the ratio $\frac{n}{c(\mathcal{X}) k(\mathcal{X})}$ taken over all non-partly-regular coherent configurations $\mathcal{X}$ of degree $n$, where $k(\mathcal{X})$ is the maximal cardinality of a fiber of $\mathcal{X}$. Clearly, $f(n)>0$. Theorem 4.1 states that $f(n)<2$. It would be interesting to find the function $f(n)$ explicitly. We have found a (schurian) non-partly-regular coherent configuration with parameters

$$
n=24, \quad k=8, \quad c=4,
$$

which shows that $f(24) \geq 3 / 4$.

\section{ORCID iDs}

Gang Chen (D) https://orcid.org/0000-0001-5173-6710

Jiawei He (D) https://orcid.org/0000-0001-7526-1246

Ilia Ponomarenko (D) https://orcid.org/0000-0003-2444-731X

Andrey Vasil'ev (D) https://orcid.org/0000-0002-6498-0138

\section{References}

[1] J. Bamberg, M. Giudici, M. W. Liebeck, C. E. Praeger and J. Saxl, The classification of almost simple $\frac{3}{2}$-transitive groups, Trans. Amer. Math. Soc. 365 (2013), 4257-4311, doi: 10.1090/s0002-9947-2013-05758-3.

[2] G. Chen and I. Ponomarenko, Coherent configurations associated with TI-subgroups, J. Algebra 488 (2017), 201-229, doi:10.1016/j.jalgebra.2017.06.004.

[3] G. Chen and I. Ponomarenko, Coherent configurations, Central China Normal University Press, 2019, http: //www.pdmi.ras.ru/ inp/.

[4] S. Evdokimov and I. Ponomarenko, Separability number and Schurity number of coherent configurations, Electron. J. Combin. 7 (2000), Research Paper 31, 33, doi:10.37236/1509.

[5] S. A. Evdokimov and I. N. Ponomarenko, Characterization of cyclotomic schemes and normal Schur rings over a cyclic group, St. Petersburg Math. J. 14 (2003), 189-221.

[6] D. G. Higman, Coherent configurations. I. Ordinary representation theory, Geometriae Dedicata 4 (1975), 1-32, doi:10.1007/bf00147398.

[7] M. Hirasaka, K.-T. Kim and J. R. Park, Every 3-equivalenced association scheme is Frobenius, J. Algebraic Combin. 41 (2015), 217-228, doi:10.1007/s10801-014-0533-6.

[8] H. D. L. Hollmann and Q. Xiang, Pseudocyclic association schemes arising from the actions of $\operatorname{PGL}\left(2,2^{m}\right)$ and PГL $\left(2,2^{m}\right)$, J. Combin. Theory Ser. A 113 (2006), 1008-1018, doi:10.1016/ j.jcta.2005.09.004. 
[9] M. Klin, C. Pech and S. Reichard, Coco2p - a gap4 package, ver. 0.18, Downloaded from: https://github.com/chpech/COCO2P/, 2020.

[10] M. W. Liebeck, C. E. Praeger and J. Saxl, The classification of $\frac{3}{2}$-transitive permutation groups and $\frac{1}{2}$-transitive linear groups, Proc. Amer. Math. Soc. 147 (2019), 5023-5037, doi:10.1090/ proc/13243.

[11] D. M. Mesner, Negative latin square designs, in: Institute of Statistics mimeo series 410, North Carolina State University. Dept. of Statistics, 1964, http: / / www . lib. ncsu . edu/ resolver/1840.4/2435.

[12] B. Moon, Every 4-equivalenced association scheme is Frobenius, Graphs Combin. 36 (2020), 401-414, doi:10.1007/s00373-019-02037-y.

[13] M. Muzychuk and I. Ponomarenko, On pseudocyclic association schemes, Ars Math. Contemp. 5 (2012), 1-25, doi:10.26493/1855-3974.121.885.

[14] M. Muzychuk and P.-H. Zieschang, On association schemes all elements of which have valency 1 or 2, Discrete Math. 308 (2008), 3097-3103, doi:10.1016/j.disc.2007.08.035.

[15] J. R. Park, On 4-equivalenced association schemes, Bull. Korean Math. Soc. 52 (2015), 16831709, doi:10.4134/bkms.2015.52.5.1683.

[16] D. S. Passman, Solvable $\frac{3}{2}$-transitive permutation groups, J. Algebra 7 (1967), 192-207, doi: 10.1016/0021-8693(67)90055-5.

[17] I. Ponomarenko and A. Vasil'ev, Cartan coherent configurations, J. Algebraic Combin. 45 (2017), 525-552, doi:10.1007/s10801-016-0715-5.

[18] A. V. Vasil'ev and D. V. Churikov, The 2-closure of a $\frac{3}{2}$-transitive group in polynomial time, Sib. Mat. J. 60 (2019), 279-290, doi:10.1134/S00374466619020083. 